This item was submitted to Loughborough's Research Repository by the author.

Items in Figshare are protected by copyright, with all rights reserved, unless otherwise indicated.

\title{
Analytical and finite element modelling of the elastic-plastic behaviour of metallic strands under axial-torsional loads
}

PLEASE CITE THE PUBLISHED VERSION

https://doi.org/10.1016/j.jimecsci.2016.06.016

PUBLISHER

(c) Elsevier

VERSION

AM (Accepted Manuscript)

\section{PUBLISHER STATEMENT}

This work is made available according to the conditions of the Creative Commons Attribution-NonCommercialNoDerivatives 4.0 International (CC BY-NC-ND 4.0) licence. Full details of this licence are available at: https://creativecommons.org/licenses/by-nc-nd/4.0/

\section{LICENCE}

CC BY-NC-ND 4.0

\section{REPOSITORY RECORD}

Foti, Francesco, and Alessandro de Luca di Roseto. 2019. "Analytical and Finite Element Modelling of the Elastic-plastic Behaviour of Metallic Strands Under Axial-torsional Loads”. figshare. https://hdl.handle.net/2134/25944. 


\section{Author's Accepted Manuscript}

Analytical and finite element modelling of the elastic-plastic behaviour of metallic strands under axial-torsional loads

Francesco Foti, Alessandro de Luca di Roseto

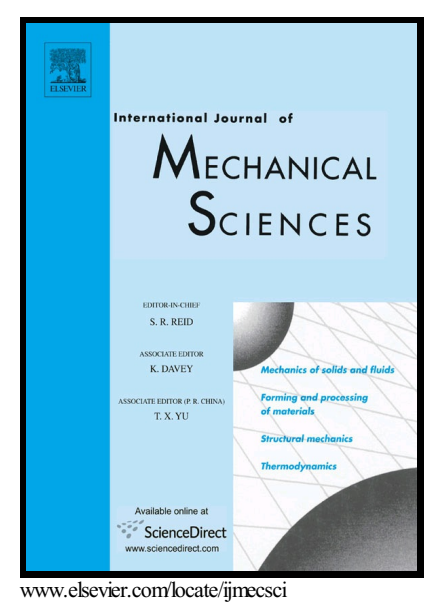

PII: $\quad$ S0020-7403(16)30103-5

DOI: $\quad$ http://dx.doi.org/10.1016/j.ijmecsci.2016.06.016

Reference: MS3328

To appear in: International Journal of Mechanical Sciences

Received date: 18 February 2016

Revised date: 20 June 2016

Accepted date: 21 June 2016

Cite this article as: Francesco Foti and Alessandro de Luca di Roseto, Analytica and finite element modelling of the elastic-plastic behaviour of metallic strand under axial-torsional loads, International Journal of Mechanical Sciences http://dx.doi.org/10.1016/j.ijmecsci.2016.06.016

This is a PDF file of an unedited manuscript that has been accepted fo publication. As a service to our customers we are providing this early version o the manuscript. The manuscript will undergo copyediting, typesetting, an review of the resulting galley proof before it is published in its final citable form Please note that during the production process errors may be discovered whic could affect the content, and all legal disclaimers that apply to the journal pertain 


\title{
Analytical and Finite Element modelling of the elastic-plastic behaviour of metallic strands under axial-torsional loads
}

\author{
Francesco Foti ${ }^{1}$ \\ Politecnico di Milano \\ Department of Civil and Environmental Engineering \\ P.zza L. da Vinci 32, Milano 20133, Italy \\ Alessandro de Luca di Roseto \\ Loughborough University \\ School of Civil and Building Engineering \\ Sir Frank Gibb Building, Loughborough LE11 3TU, England, United Kingdom
}

\begin{abstract}
In this work a new formulation for modelling the elastic-plastic behaviour of metallic strands subjected to axial-torsional loads is presented. Simple and accurate cross sectional constitutive equations are derived, fully accounting for the evolution of plastic deformations in the wires, starting from a description of the internal structure of the strand. The proposed approach is suitable both for straightforward analytical calculations as well as for implementation into finite elements for the large-scale structural analyses of cable structures. A full three-dimensional (3D) finite element (FE) model, based on a parametric description of the strand internal geometry, is also developed. The results of both the FE model and the analytical formulation are validated with reference to a well documented physical testing campaign and a well-established linearly elastic literature model. Additional analyses are then performed to carefully assess the validity of the proposed mechanical formulation, for a wide range of strand construction parameters, by means of systematic comparisons against the results of the 3D FE model and of a recent linearly elastic literature model.
\end{abstract}

Email addresses: francesco.foti@polimi.it (Francesco Foti), a.delucadiroseto@lboro. ac .uk (Alessandro de Luca di Roseto)

${ }^{1}$ Corresponding author 
Keywords: Strands, axial-torsional loads, elastic-plastic behaviour, finite element method, curved thin rod theory

\section{Introduction}

Metallic strands are made of helical wires, twisted around a straight core and grouped in concentric layers. The core is typically another wire (core wire), which contributes to sustain the external loads and provides a radial support to the layers. The

5 simplest construction consists of a single layer surrounding the core. Six outer wires are usually employed in this basic case, which will be referred in the following as simple strand. A review of the most common strand typologies, including details on their internal geometry, mechanical properties and manufacturing process, can be found e.g. in [9].

Due to their ability in carrying large axial forces with relatively small dead-loads, metallic strands are efficient structural members, widely employed in mechanical and civil engineering applications. Moreover, they can be helically wound to form wire ropes, which are used e.g. in hoisting devices, tethered marine structures, suspended bridges. A distinctive feature of strands is the coupling between the axial and torsional behaviour, due to the helicoidal geometry of the wires.

Many studies have been devoted to investigate the response of strands under a combination of axial force and torsional moment (detailed reviews can be found e.g. in: [3], [10]). Most of them rely on the assumption of linearly elastic wires, thus providing an insight into the behaviour of strands under service loads. Within this context, analytical formulations (see e.g. [4]) have been developed to estimate both the stress state of wires as well as global response parameters, such as the coupled axial-torsional cross sectional stiffness matrix. Only few works, instead, considered the evolution of plastic deformations under axial-torsional loads. Jiang et al. ([13], [14], [15]) proposed a refined finite element (FE) approach, which takes into account plastic deformations and allow for an accurate description of internal contact conditions. More recently, procedures for developing full three-dimensional (3D) elastic-plastic FE models of metallic strands have been proposed e.g. by Judge et al. [17] and by Yu et al. [30], while 
Imrak and Erdönmez [12] adopted the FE method to study wire ropes with complex cross sections under the assumption of elastic-plastic material behaviour. Due to their huge computational cost, however, rich FE models cannot be successfully applied for simple calculations or large-scale structural analyses, which are typical of engineering applications. As a consequence, the post-elastic behaviour of cable structures is typically investigated by considering simple phenomenological uni-axial constitutive laws (e.g.: [16], [18], [19]), which don't take into account the peculiar internal structure of strands and the direct consequences on the mechanical response, such as axial-torsional coupling.

To overcome these limitations, a new approach is presented in this work to model the elastic-plastic behaviour of simple strands subjected to axial-torsional loads. Cross sectional constitutive equations are derived starting from a description of the internal structure of the strand, herein considered as a composite structural element, and fully accounting for plasticity effects. The aim is to provide for a sound mechanical framework, suitable for both simple analytical calculations as well as implementation into finite elements for the large-scale structural analyses. Each wire of the strand is individually modelled as a curved thin rod in the framework of the classic Kirchhoff-ClebschLove theory [25]. The von Mises yield criterion is adopted, to model the interaction between normal and tangential stresses on the wire cross sections, together with the well-known Prandtl-Reuss associated flow rule. Kinematic equations are introduced to relate the axial strain and the torsional curvature of the strand to the generalized strains of the wires. Then, starting from the knowledge of the normal and tangential stress distributions over the cross sections of the wires, the resultant axial force and torsional moment of the strand are evaluated with equilibrium considerations.

The proposed mechanical formulation is first directly assessed with experimental results from the literature, secondly, with the development of a full 3D FE model of the strand, which allowed to carefully investigate the performance of the proposed formulation for a wide range of strand construction parameters.

The results of both the analytical as well as the FE model are preliminary validated with reference to a well documented benchmark. Comparisons are carried out with respect to both available experimental results [27] and a well-established linearly elastic 
literature model [4]. Additional analyses are then performed, for different strand constructions, to check the accuracy of the proposed elastic-plastic analytical formulation by means of systematic comparisons against the results of the 3D FE model and of a recent linearly elastic literature model.

\section{Geometry of the strand}

A simple strand made of seven wires with circular cross section is considered in this work, as depicted in Figure 1. The geometry of the internal structure is described with reference to the straight configuration of the strand. To this aim, a right-handed Cartesian system, with axes $\left\{x_{i}\right\}$ and unit vectors $\left\{\mathbf{e}_{i}\right\} \quad(i=1,2,3)$, is defined such that $x_{1}$ coincides with the strand centerline. A generic wire, then, is represented as a curved thin rod, by specifying for each cross section the position of the centroid and the orientation with respect to the axes $\left\{x_{i}\right\}$.

The centerline of the external wires is described through circular helices, with radius $R$ and pitch $P$, by means of position vectors with the following form:

$$
\mathbf{x}_{w}(\theta)=\frac{P}{2 \pi}\left(\theta-\theta_{0}\right) \mathbf{e}_{1}+R \cos (\theta) \mathbf{e}_{2}+R \sin (\theta) \mathbf{e}_{3}
$$

The symbol $\theta$ in (1) denotes the swept angle, i.e. the angle which the projection of the position vector on the plane $x_{1}=0$ defines with the axis $x_{2}$. The subscript " 0 " is adopted to identify the value of the swept angle at $x_{1}=0$.

The orientation of the wire cross sections, then, can be described by specifying a local system of axes, attached to the helicoidal centerline. To this aim, the right-handed Serret-Frenet unit vectors $\left\{\mathbf{f}_{i}(\boldsymbol{\theta})\right\} \quad(i=1,2,3)$ are introduced, such that: $\mathbf{f}_{1}(\boldsymbol{\theta})$ is the tangent vector, while $\mathbf{f}_{2}(\theta)$ and $\mathbf{f}_{3}(\theta)$ are, respectively, the normal and binormal unit vectors of the wire centerline. The Serret-Frenet unit vectors can be evaluated starting from (1) (see e.g. [21]) and related to the unit vectors $\left\{\mathbf{e}_{i}\right\}$ by means of a rotation tensor, $\boldsymbol{\Lambda}_{w}(\boldsymbol{\theta})$, i.e: $\mathbf{f}_{i}(\boldsymbol{\theta})=\boldsymbol{\Lambda}_{w}(\boldsymbol{\theta}) \mathbf{e}_{i},(i=1,2,3)$. By denoting as $\alpha$ the lay angle of the wire, i.e. the constant angle which the tangent vector $\mathbf{f}_{1}$ defines with the strand axis $x_{1}$, the components of $\boldsymbol{\Lambda}_{w}(\theta)$ with respect to the basis $\left\{\mathbf{e}_{i}\right\}$ (here denoted as $\left[\boldsymbol{\Lambda}_{w, i j}\right]$ ) 
can be expressed as follows:

$$
\left[\boldsymbol{\Lambda}_{w, i j}\right]=\left[\begin{array}{ccc}
\cos (\alpha) & 0 & \sin (\alpha) \\
-\sin (\alpha) \sin (\theta) & -\cos (\theta) & \cos (\alpha) \sin (\theta) \\
\sin (\alpha) \cos (\theta) & -\sin (\theta) & -\cos (\alpha) \cos (\theta)
\end{array}\right]
$$

Starting from equation (1), the initial curvature $(\kappa)$ and torsion $(\tau)$ of the wire centerline, which are of special importance in their mechanical modelling, can be defined, respectively, as: $\kappa=\frac{\sin ^{2}(\alpha)}{R}$ and $\tau=\frac{\sin (\alpha) \cos (\alpha)}{R}$ (see e.g. [21]). It's worth noting that the lay angle can be related to the helix radius and pitch through the simple geometric relation: $\alpha=\tan ^{-1}\left(\frac{2 \pi R}{P}\right)$. Hence, the geometry of the external wires can be completely defined by specifying two construction parameters only, namely: the helix radius $R$ and the pitch $P$ (or equivalently the lay angle $\alpha$ ).

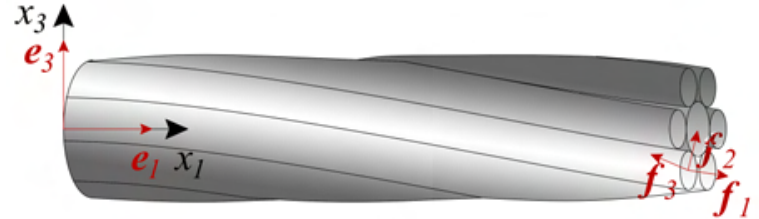

(a)

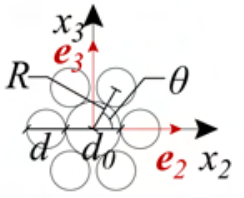

(b)

Figure 1: Geometry of the strand. (a) Side view. (b) Cross section. The effect of the lay angle on the projection of the cross sections of the wires is neglected in Figure 1(b).

Two basic internal contact modes can be distinguished (see also [3]): radial contact (Figure 2(a)), and lateral (or circumferential) contact (Figure 2(b)). In the first case the external wires are in contact with the core, but not among them. Accordingly, the helix radius $R$ is simply given by the sum of the diameters of the core and of the external wires, i.e.: $R=\frac{1}{2}\left(d_{0}+d\right)$. In the lateral contact case, instead, the external wires are in contact with their neighbours, but not with the core wire. The helix radius, hence, turns out to be independent of the wire core diameter and can be evaluated as: ${ }_{100} R=\frac{d}{2} \sqrt{1+\frac{3}{\cos ^{2}(\alpha)}}$, by assuming that wire cross sections are elliptical in a plane normal to the strand centerline [4].

It's worth observing, however, that typical strand constructions are characterized by clearances among the external wires, in order to reduce interwire frictional effects 
and secondary tensile stresses which can arise whenever the strand is bent ([4], [9]). As a consequence, a purely radial contact mode is assumed in this work to define a "reference" geometric framework for the mechanical modelling of the strand, with both analytical as well as FE techniques. This hypothesis amounts to consider: (a) a core wire with greater diameter than the external wires, and (b) lay angles smaller than a maximum value, $\alpha_{\max }$, corresponding to the onset of lateral contact. The lay angle $\alpha_{\max }$ can be easily evaluated by imposing that the helix radius $R$ simultaneously satisfies the geometric conditions for the radial and lateral contact previously introduced. After some straightforward calculations, the following geometrical condition can be obtained:

$$
\alpha<\alpha_{\max }=\arccos \left(\sqrt{\frac{3}{\left(1+\frac{d_{0}}{d}\right)^{2}-1}}\right), \text { with }: \frac{d_{0}}{d}>1
$$

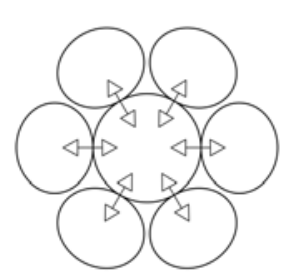

(a)

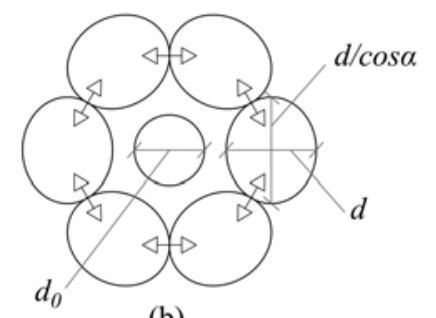

(b)

Figure 2: Cross section of the strand. Definition of contact modes: (a) radial contact; (b) lateral contact.

\section{The elastic-plastic mechanical model}

Let us consider a strand free from constraints, straight in the reference (undeformed) configuration and subjected to constant axial force, $F_{s}$, and torsional moment, $M_{s}$ (see Figure 3(a)). Due to the symmetry with respect to the strand centerline (axis $\left.x_{1}\right)$ and the imposed boundary conditions, the element undergoes constant axial strain, $\varepsilon_{s}$, and torsional curvature, $\chi_{s}$. These generalized strain variables are conjugated to the 
cross sectional stress resultants, $F_{S}$ and $M_{s}$, in the expression of the internal work per unit length of the strand. A generalized axial-torsional constitutive law for the strand cross section, hence, can be formally stated through the functions: $F_{s}=F_{s}\left(\varepsilon_{s}, \chi_{s}\right)$ and $M_{s}=M_{s}\left(\varepsilon_{s}, \chi_{s}\right)$. The latter are derived in this section starting from a description of the mechanical behaviour of wires, herein regarded as curved thin rods made of elasticplastic material. Kinematic equations are introduced to relate the strains of the wires to the generalized strain variables of the strand and a procedure for the evaluation of the stress distribution over the wire cross section is outlined. Then, the strand cross sectional stress resultants $F_{S}$ and $M_{S}$ are defined through equilibrium considerations.

The wires can be modelled within the framework of the Kirchhoff-Clebsch-Love theory for curved thin rods [25]. Accordingly, shear deformability is neglected and the cross sections are assumed to remain plane and normal to the wire centerline. Under the further assumption of small displacements and strains, the mechanical response of the wires can be studied within a local reference frame, attached to the helicoidal centerline and with axes directed as the Serret-Frenet unit vectors $\left\{\mathbf{f}_{i}\right\}$ introduced in Section 2 ([7], [8]). Within this context, the generalized stresses of the curved thin rod model are the axial force, $F_{w 1}$, and the moments acting with respect to the directions of the vectors $\left\{\mathbf{f}_{i}\right\}$, i.e.: the torsional moment $M_{w 1}$, and the bending moments $M_{w 2}$ and $M_{w 3}$ (see Figure 3(b)). The work-conjugated strain measures are the wire axial strain $\varepsilon_{w}$ (i.e. the elongation of the wire centerline) and the mechanical curvatures $\chi_{w i}(i=1,2,3)$ with respect to the axes of the local, wire-attached, reference system. The mechanical curvatures are here introduced according to the definition provided in [8] and reported in Appendix A (see eq. (A.1)).

The same mechanical model is also adopted to describe the behaviour of the straight core, with minor modifications: in the case of the core, indeed, the unit vectors $\left\{\mathbf{f}_{i}\right\}$ of the local reference frame simply coincide with those of the strand reference system $\left\{\mathbf{e}_{i}\right\}$.

The axial strain of the wires, $\varepsilon_{w}$, can be evaluated by exploiting classic literature results. In fact, several authors (e.g.: [20], [23], [4]) have shown that, during the axial-torsional loading of the strand: (a) the core is subjected to the same elongation of the strand, and (b) the external wires undergo a transformation which preserves 


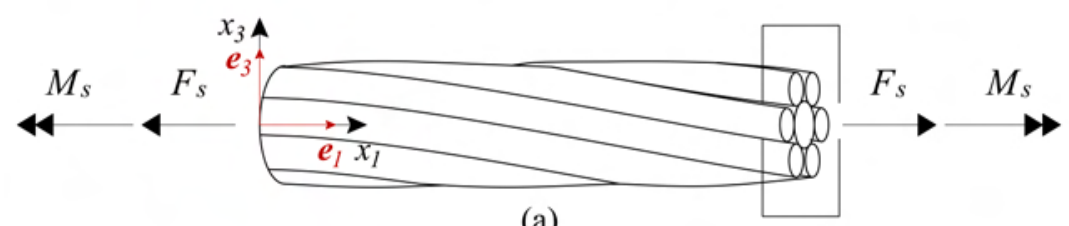

(a)

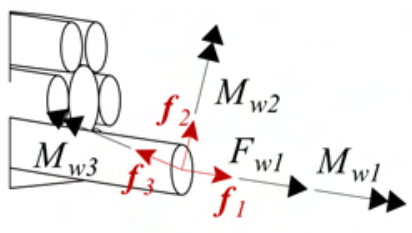

(b)

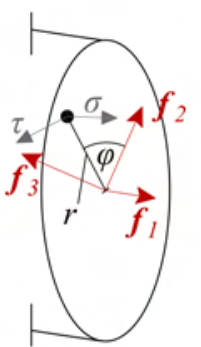

(c)

Figure 3: (a) Straight strand subjected to axial-torsional loads. (b) Generalized stresses on the wire cross section. (c) Normal $(\sigma)$ and tangential $(\tau)$ stress components on the wire cross section.

the geometrical shape of their centerline. Accordingly, the deformed centerline of the external wires is a circular helix, possibly characterized by different radius and pitch with respect to the reference configuration. Variations of the helix radius can be due to: (1) the contraction of the diameters of both the external and the core wires due to the Poisson effect, and (2) the deformation of the internal contact surfaces between wires and core (wire flattening). These phenomena can significantly influence the response of large-diameter strands, but can be practically neglected for the very common case of simple strands with a steel core [26]. Starting from this latter hypothesis, Lanteigne [23] derived the following kinematic equation:

$$
\varepsilon_{w}=\cos ^{2}(\alpha) \varepsilon_{s}+R \sin (\alpha) \cos (\alpha) \chi_{s}
$$

The mechanical curvatures $\chi_{w i}(i=1,2,3)$ can be evaluated, under the assumption that the wire cross section rigidly rotates with the cross section of the strand, by tailoring to the case at study the general kinematics equations first derived in [8]. Skipping the calculations, which are fully reported in Appendix A, the following expressions are 
obtained:

$$
\chi_{w 1}=\cos ^{2}(\alpha) \chi_{s} ; \chi_{w 2}=0 ; \chi_{w 3}=\sin (\alpha) \cos (\alpha) \chi_{s}
$$

By considering a lay angle equal to zero, equations (4) and (5a, b, c) hold true for the core wire also, which thus undergoes to the same combination of axial strain and torsional curvature as per the strand element. On the other hand, due to their initial curved configuration, the external wires are in general subjected to a more complex strain state, characterized by a combination of axial elongation, torsion and bending about the binormal unit vector of the Serret-Frenet triad $\left(\mathbf{f}_{3}\right)$. It's also worth noting that equations (4) and (5a, b, c) do not depend on the swept angle $\theta$, introduced in Section 2. As a consequence, all external wires behave identically and the generalized strain variables are constant along their length. It can be concluded, hence, that the proposed kinematic model satisfies the symmetry with respect to the strand centerline (axis $x_{1}$ ), known to characterize the mechanical problem under study.

Focusing now on the wire cross section and recalling the hypothesis of small strains, the normal $(\varepsilon)$ and tangential $(\gamma)$ strain components at a generic point, identified by the polar coordinates $(r, \varphi)$ defined in Figure (3(c)), can be expressed as:

$$
\varepsilon(r, \varphi)=\varepsilon_{w}-r \cos (\varphi) \chi_{w 3} ; \gamma(r, \varphi)=r \chi_{w 1}
$$

In order to introduce an elastic-plastic constitutive law, the strain components $\varepsilon$ and $\gamma$ can be additively decomposed into purely elastic $\left(\varepsilon_{e}, \gamma_{e}\right)$ and plastic $\left(\varepsilon_{p}, \gamma_{p}\right)$ contributions, such that: $\varepsilon=\varepsilon_{e}+\varepsilon_{p}$ and $\gamma=\gamma_{e}+\gamma_{p}$. Accordingly, the normal $(\sigma)$ and tangential stresses $(\tau)$ depicted in Figure 3(c) are related to the elastic strain components only. Under the assumption of homogeneity and isotropy of material and denoting respectively as $E$ and $v$ the Young modulus and the Poisson coefficient, the following equations are introduced:

$$
\sigma=E \varepsilon_{e} ; \quad \tau=\frac{E}{2(1+v)} \gamma_{e}
$$

Consistently with numerical and experimental literature studies on the axial-torsional behaviour of simple strands made of cold-drawn steel components ([13], [17], [27]): 
(a) the elastic domain (herein denoted through the function: $F(\sigma, \tau)$ ) is modelled by adopting the von Mises yield criterion (Eq. (8a)), along with an isotropic linear hardening law (Eq. (8b)), and (b) the evolution of plastic strains is described though the classic Prandtl-Reuss associated flow rule (Eq. (9)), herein introduced in rate form by denoting with a dot the derivative with respect to a time-like variable:

$$
\begin{gathered}
F(\sigma, \tau)=\sqrt{\sigma^{2}+3 \tau^{2}}-\sigma_{y}(\lambda) \leq 0 \\
\sigma_{y}(\lambda)=\sigma_{y 0}+\lambda \frac{E^{\prime}}{1-\frac{E^{\prime}}{E}} \\
\left\{\begin{array}{l}
\dot{\varepsilon}_{p}=\dot{\lambda} \frac{\partial F}{\partial \sigma} \\
\dot{\gamma}_{p}=\dot{\lambda} \frac{\partial F}{\partial \tau}
\end{array}\right.
\end{gathered}
$$

The symbols $\sigma_{y 0}$ and $E^{\prime}$ denote, respectively, the first-yielding stress and the postyielding Young modulus of the material (see the bilinear uni-axial stress-strain curve

$$
\dot{\lambda} \geq 0, \dot{\lambda} F=\dot{\lambda} \dot{F}=0
$$

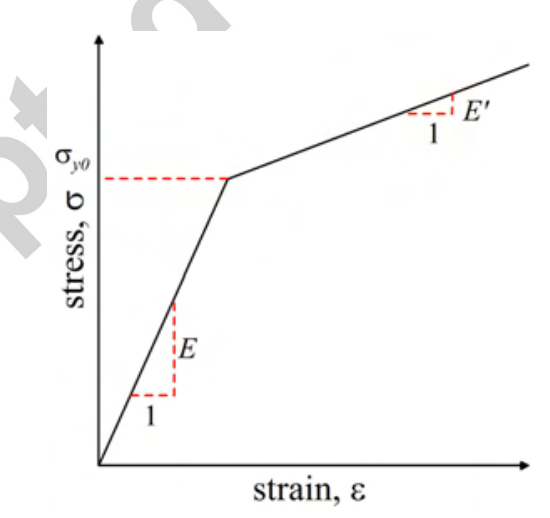

Figure 4: Bi-linear elastic-plastic stress-strain curve of the material.

The resultant axial force, $F_{w 1}$, and moments, $M_{w i}(i=1,2,3)$, of the wire (see also 
Figure 3(b)), are then evaluated through integration over the cross sectional area $A_{w}$ :

$$
\begin{gathered}
F_{w 1}=\int_{A_{w}} \sigma d A_{w} ; M_{w 1}=\int_{A_{w}} r \tau d A_{w} \\
M_{w 2}=\int_{A_{w}} r \sin (\varphi) \sigma d A_{w} ; M_{w 3}=-\int_{A_{w}} r \cos (\varphi) \sigma d A_{w}
\end{gathered}
$$

Due to the already mentioned symmetry of the mechanical model with respect to 200 the strand centerline, all external wires are characterized by the same cross sectional stress resultants (11). This leads to a significant simplification in the evaluation of the strand cross sectional resultants, which, fully accounting for the projection from the reference system of the wire to the one of the strand (see: Section 2, eq. (3)), can be expressed as:

$$
\begin{aligned}
& F_{s}=F_{0}+6 \cos (\alpha) F_{w 1} \\
& M_{s}=M_{0}+6\left(\cos (\alpha) M_{w 1}+\sin (\alpha) M_{w 3}\right)+6 \sin (\alpha) R F_{w 1}
\end{aligned}
$$

where $F_{0}$ and $M_{0}$ are, respectively, the axial force and the torsional moment of the core wire.

The equations from (4) to (12) completely define the elastic-plastic axial-torsional behaviour of the strand cross section. They allow for the direct evaluation of $F_{S}$ and $M_{s}$, if the evolution in time of the strand axial strain and torsional curvature is known. For assigned values of the axial force and torsional moment, on the other hand, the set of cross sectional constitutive equations can be iteratively solved for $\varepsilon_{s}$ and $\chi_{s}$. To this aim, a Newton-Raphson solver is adopted in this work along with a classic one-step backward Euler algorithm (see e.g. [5]) to integrate the rate-form elastic-plastic constitutive equations (8)-(10) over a regular grid of concentric control points on the wire cross section. The density of the grid is controlled by specifying in the polar coordinates of the wire $(r, \varphi)$ the radial and angular discretization intervals: $\Delta r$ and $\Delta \varphi$. The stresses at the control points are then used to evaluate the wire stress resultants, defined in equations (11a, b, c, d), by means of a standard numerical integration technique. 


\section{The finite element model}

A full three-dimensional (3D) finite element (FE) model has been developed using the ANSYS 15.0 software, to simulate the axial-torsional loading of straight strands. The aim is to provide an accurate numerical tool for a comparative assessment of the mechanical formulation presented in Section 3.

The FE approach herein proposed relies on the parametric description of the internal geometry provided in Section 1, which allows to easily account for variations of the strand construction parameters. A solid model of the strand is first created in SolidWorks 2013 CAD, by extruding the round cross sections along the helix wire centerlines defined through equation (1). A linear extrusion along the strand centerline, instead, is adopted for the core wire. A purely radial contact mode is considered among the external wires and the core, as it is usual in typical strand constructions and consistently with the mechanical model presented in Section 3. As a consequence, the core diameter is assumed to be greater than the one of the external wires and the lay angle $\alpha$ is always smaller than the maximum value $\alpha_{\max }$ defined in (3).

The 3D geometric model of the strand is then imported in ANSYS for the mesh generation and the definition of the mechanical properties of the finite element model. Twenty-node brick element with quadratic displacement behaviour (SOLID 186 [1]) are adopted, along with the elastic-plastic constitutive law already described in Section 3. Preliminary mesh sensitivity analyses have been carried out to identify an appropriate mesh density [6]. A good trade-off between accuracy of results and computational cost is found by setting the maximum size of the elements equal to $1 / 5$ of the wire diameter in the radial direction, and to $1 / 40$ of the lay length in the longitudinal direction. A medium intensity "smoothing" is also used to reduce mesh distortion [1]. Examples of the FE mesh are shown in Figure 5 for different values of lay angle $\alpha$.

Surface-to-surface contact pairs are used to model the internal contact among wires, accounting for both deformability of contact surfaces as well as possible relative sliding between wires. Friction is modelled through the classic Coulomb law. Preliminary tests, however, have shown that the global response of the FE strand model to axial-torsional loads is not affected in practice by the value of the interwire friction 
coefficient [6]. The latter finding is in good agreement with other literature results (see e.g. [10]). Each contact pair is modelled with CONTA 174 and TARGE 170 elements, while an Augmented Lagrangian algorithm is adopted to enforce contact compatibility conditions. The latter has been preferred to a Pure Penalty algorithm, in order to minimize the sensitivity of the solution to the value of the normal contact stiffness [1].

Two rigid surface-based constraints are created at the end sections of the strand, to couple the motion of all the wire and core nodes to that of a single master node. The creation of these constraints greatly simplify the definition of the boundary conditions, which can be completely characterized by imposing a generalized force (axial force or torsional moment) or displacement (axial displacement or torsional rotation) to the master node.

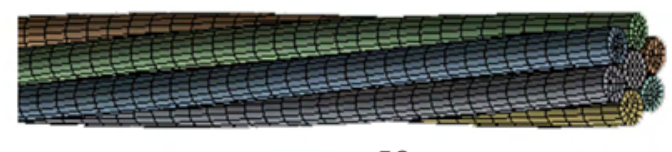

$$
\alpha=5^{\circ}
$$
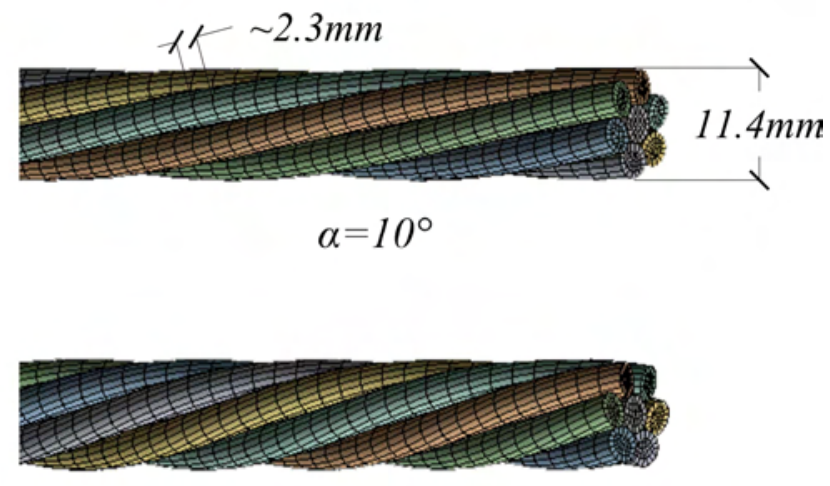

$$
\alpha=15^{\circ}
$$

Figure 5: Examples of 3D FE meshes for different values of the lay angle $\alpha$. 


\section{Experimental validation and numerical applications}

Both the elastic-plastic mechanical model herein proposed, as well as the finite element (FE) approach we propose in this work have been validated with reference to a well-documented benchmark, widely studied in literature with both analytical as well as numerical techniques (e.g.: [28], [13], [17]) and for which reliable experimental data are available [27]. The experimental tests were performed by means of a tensiontorsion machine, which can apply a prescribed axial elongation to a straight strand specimen, while measuring the corresponding axial force. One end of the strand is fully clamped, whereas the other one can be free to rotate with respect to the strand centerline (free-end case) or fully restrained against rotations (fixed-end case). In the latter case, the reacting torque is measured to quantify the coupling between axial and torsional behaviour.

The testing procedure allows to simulate the theoretical condition of constant axial force and torsional moment along the specimen, which can be very conveniently studied by means of the proposed analytical cross sectional model. Different sets of control points on the wire cross sections, with populations ranging from $25(\Delta r=d / 10$, $\left.\Delta \varphi=60^{\circ}\right)$ to $649\left(\Delta r=d / 20, \Delta \varphi=5^{\circ}\right)$ points, have been preliminary tested, showing a very fast convergence of the integration technique adopted to evaluate the wire stress resultants defined in equations $(11 \mathrm{a}, \mathrm{b}, \mathrm{c}, \mathrm{d})$. In this section, the results obtained with the most refined grid of integration points will be shown, in order to present a very detailed picture of the local stress distributions predicted by the proposed mechanical formulation.

Finite element simulations, instead, are performed by modelling a stretch of strand with a length of $500 \mathrm{~mm}$, corresponding to about five times the lay length of the external wire, to minimize the influence of boundary conditions (end effects) on the solution. A mesh of about 28000 elements and 137400 nodes is defined according to the criteria presented in Section 4 (see also Figure 5). The geometric and material parameters of the strand are taken from [13] and listed in Table 1. The results of the proposed analytical and FE models will be compared with both the experimental results from [27] as well as the predictions of a well-known theory developed by Costello [4]. 
Differently than the mechanical model proposed in this paper, Costello's one is based on the hypothesis of linearly elastic material behaviour. On the other hand, the geometric nonlinearities due to the radial contraction of the external and core wires (Poisson effect), and the related changes in the strand internal structure, are fully accounted for. The model is geometrically non linear, linearized expressions have been proposed e.g. in [29], [22]. The fully non-linear Costello's model, however, is at the base of the solution presented by Jiang et al. [13] and reported in this paper.

A comparison among theoretical and experimental results is shown in Figure 6. The proposed analytical and FE models are in excellent agreement with the experimental data and allow to capture, both from a qualitative as well as from a quantitative point of view, the non-linear evolution of the axial load, which is represented in Figure 6(a) as a function of the axial strain $\varepsilon_{s}$. Also the coupling between the axial force and the torsional moment, experimentally determined through the fixed-end test, is well represented by the proposed models, as it can be observed from Figure 6(b). The relation between the axial force and torsional moment delivered from both the analytical and the FE model is almost linear, with a small change of slope corresponding to the complete plasticization of the strand cross section. The latter condition can be easily identified through the abrupt change of slope of the fixed-end curve in Figure 6(a).

Very good agreement is also observed among the proposed models and the one by Costello over the whole elastic range of the strand response. Due to the assumption of linearly elastic material, however, Costello's model obviously fails to reproduce the non-linear evolution of the axial force, which is governed by the progressive plastic deformation of wires. Table 2 shows a comparison among the elastic theoretical and experimental values of: (a) the direct axial stiffness $k_{1}=\frac{F_{s}}{\varepsilon_{s}}$, and (b) the coupling parameter $k_{2}=\frac{F_{s}}{M_{s}}$. All values listed in Table 2 have been obtained from the initial slope

Table 1: Geometric and material parameters from [13].

\begin{tabular}{ccccccc}
\hline$d_{0}(\mathbf{m m})$ & $d(\mathbf{m m})$ & $\alpha(\mathbf{m m})$ & $E(\mathbf{G P a})$ & $E^{\prime}(\mathbf{G P a})$ & $\sigma_{y 0}(\mathbf{G P a})$ & $v$ \\
\hline 3.94 & 3.73 & 11.80 & 188 & 188 & 1.54 & 0.3 \\
\hline
\end{tabular}


of the response curves (Figures 6(a) and 6(b)), with the only exception of those referred to the proposed analytical model, which can be evaluated through simple closed-form expressions (fully reported in Appendix B). Differences with respect to the experimental values of parameters $k_{1}$ and $k_{2}$ are less than $4 \%$ for all theoretical models. In particular, it can be noticed that the proposed analytical formulation shows the same accuracy of Costello's one in predicting the direct axial stiffness of the strand, both in the fixed- as well as in the free-end case. Costello's theory, on the other hand, delivers a slightly more accurate prediction of the coupling parameter $k_{2}$. This can be explained since Costello's model, accounting for the radial contraction of the wire diameters due to the Poisson effect, is based on a better estimation of the helix radius of the external wires. The latter can influence the parameter $k_{2}$ more than $k_{1}$, as it can be also inferred from inspection of the closed-form expressions given in Appendix B. A comparison between the values of $k_{2}$ obtained through the proposed analytical model and Costello's one, hence, allows to quantify the error introduced in the strand mechanical modelling by neglecting the Poisson effect, which is less than $2 \%$ for the case at study.
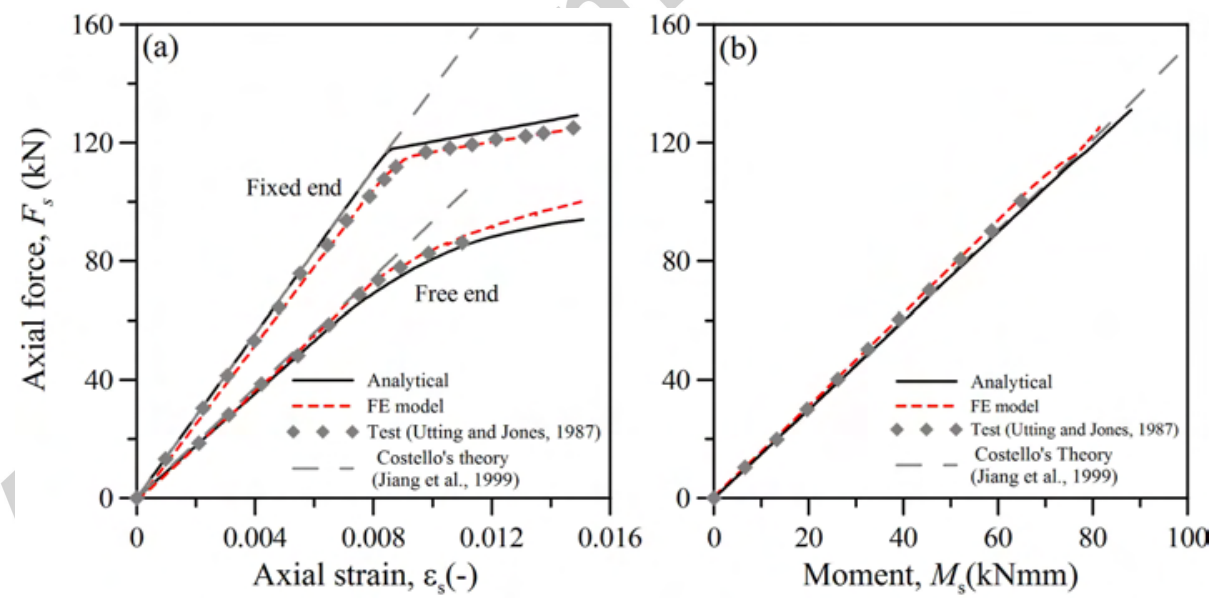

Figure 6: Comparison among theoretical and experimental results: (a) axial force vs. axial strain; (b) axial force vs. torsional moment (fixed-end test).

The effects of different torsional boundary conditions on the axial-torsional behaviour of the strand can be clearly appreciated from the load-strain curves depicted in Figure 6(a). In fact, both the initial (elastic) stiffness as well as the load-bearing capac- 
Table 2: Comparison among theoretical and experimental values of the elastic response parameters: $k_{1}=\frac{F_{s}}{\varepsilon_{s}}$, and $k_{2}=\frac{F_{s}}{M_{s}}$.

$\begin{array}{cccc}\text { Proposed Analytical } & \text { Proposed FE } & \text { Costello's Analytical } & \text { Experimental } \\ \text { Model } & \text { Model } & \text { Model } & \text { Data }\end{array}$

\begin{tabular}{ccccc} 
& Model & Model & Model & Data \\
\hline Fixed-end & & & & \\
$k_{1}=\frac{F_{s}}{\varepsilon_{s}}(\mathrm{kN})$ & 13853 & 13017 & 13835 & 13539 \\
Error $(\%)$ & 2.32 & 3.86 & 2.19 & - \\
$k_{2}=\frac{F_{s}}{M_{s}}(1 / \mathrm{mm})$ & 1.496 & 1.526 & 1.520 & 1.530 \\
Error $(\%)$ & 2.25 & 0.26 & 0.65 & - \\
Free-end & & & & \\
$k_{1}=\frac{F_{s}}{\mathcal{E}_{s}}(\mathrm{kN})$ & 8895 & 8775 & 9329 & 9140 \\
Error $(\%)$ & 2.68 & 3.9 & 2.07 & - \\
\hline
\end{tabular}

ity of the strand are significantly increased by preventing the torsional rotation of the end sections (fixed-end case). This can be easily explained through a closer analysis of the local stress state of the wires.

In the fixed-end case, the torsional curvature of the strand is equal to zero $\left(\chi_{s}=0\right)$ and, according to the proposed analytical model, all wires of the strand are simply stretched (see equations (4) and (5)) and subjected to a uniform distribution of normal stresses $(\sigma)$ over their cross sections. All points of a generic wire cross section, hence, reach the first yielding stress simultaneously. This leads to a sharp transition from the elastic to the post-elastic branch of the axial-strain response curve of the strand (see Figure 6(a)). Within this context, the load-bearing capacity of wires is fully exploited to sustain the applied axial load. Special care, however, must be paid to ensure that the end constraints are able to resist the coupling reacting moment which arises because of the peculiar internal structure of the strand (Figure 6(b)). The conclusions drawn from the analytical model are in very good agreement with the results of the more refined FE model. The normal stresses $(\sigma)$ and the Von Mises equivalent stresses $\left(\sigma_{e q}\right)$ are shown, respectively, in Figures 7(a) and 7(b) for an axial load of $120 \mathrm{kN}$, in the post-elastic region of the strand response. The normal stresses are substantially constant over the 


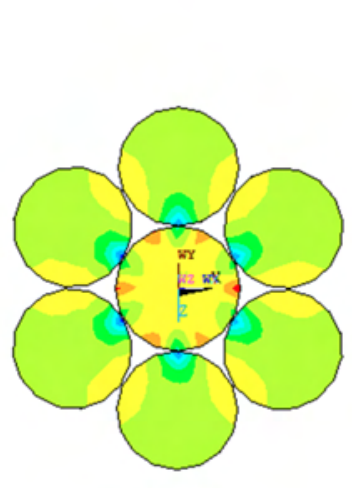

(a)

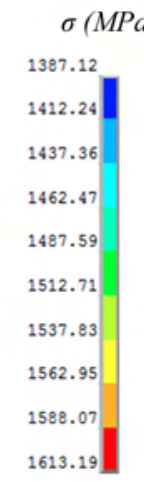

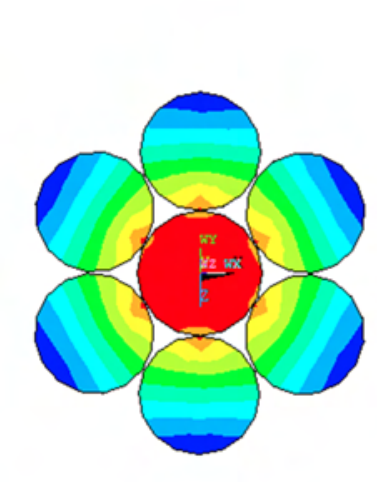

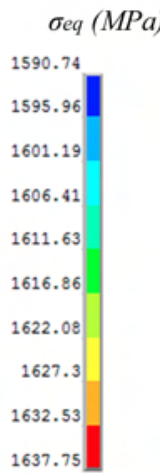

(b)

Figure 7: Fixed-end case. Results of the FE model. Load 120 kN. (a) Normal stresses. (b) Von Mises equivalent stresses.

wire cross sections, with small variations due to the normal contact pressure exerted by the external wires on the core. Contact stresses lead to an initial localization of yielding in the neighbourhood of internal contact surfaces, which, however, only slightly affect the global strand behaviour (see Figures 6(a) and 6(b)).

The application of the axial load in the free-end case, on the other hand, generates a complex and highly non-uniform stress state over the wire cross sections. Figures (8) to (11) show the normal $(\sigma)$ and Von Mises $\left(\sigma_{e q}\right)$ stress distributions obtained through the proposed analytical (Figures (8) and (9)) and FE (Figures (10) and (11)) models for the two different values of axial force: $F_{S}=21.5 \mathrm{kN}$ and $F_{S}=100 \mathrm{kN}$, which are representative, respectively, of the elastic and post-elastic region of the strand response. 360 According to the proposed analytical model (see equations (4) and (5)), the core wire is subjected to the same axial strain and torsional curvature of the strand, while the external wires undergo a combination of axial elongation, torsion and bending about the binormal unit vector of the Serret-Frenet triad $\left(\mathbf{f}_{3}\right)$. Figures 8(a) and 8(b) show the corresponding elastic distributions of the normal and Von Mises stresses, which are in good agreement with the ones evaluated through the FE model and depicted in Figures 10 (a) and 10(b). Since in the free-end case a portion of the external work done by the axial load $F_{S}$ is spent to twist and bend the wires, the initial (elastic) stiffness of the 


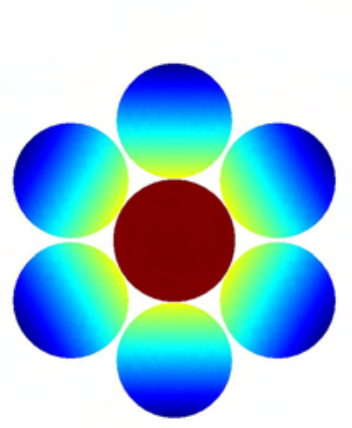

(a)

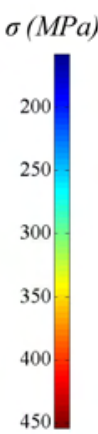

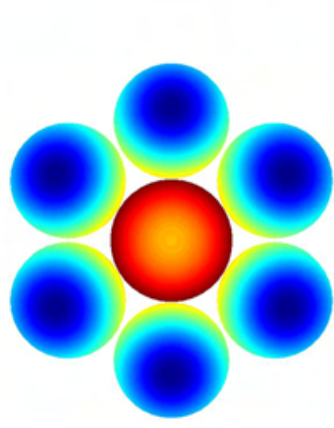

(b)

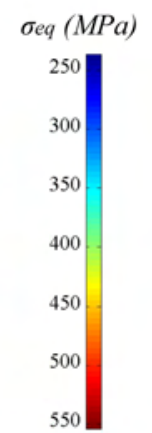

Figure 8: Free-end case. Results of the analytical model. Load 21.5 kN. (a) Normal stresses. (b) Von Mises equivalent stresses.

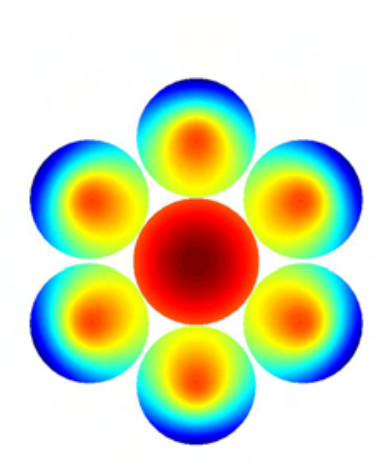

(a)

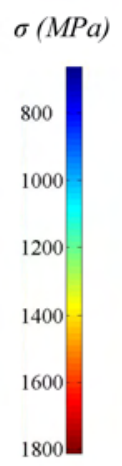

ra $\square=$

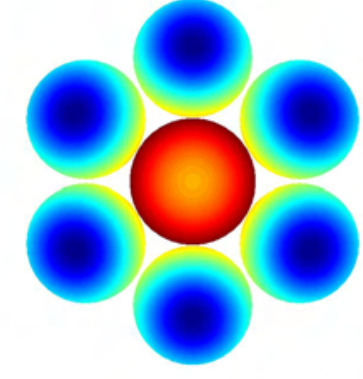

(b)

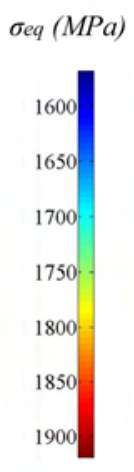

Figure 9: Free-end case. Results of the analytical model. Load 100 kN. (a) Normal stresses. (b) Von Mises equivalent stresses.

strand is lower than in the fixed-end case.

Plastic flow under the combined action of normal and tangential stress components, then, starts from the points where the Von Mises equivalent stresses are highest and gradually spreads over the wire cross sections, thus leading to a smooth transition from the elastic to the elastic-plastic response, as it can be clearly appreciated from Figure 6(a). Analytical and FE stress distributions compare quite well also in the elasticplastic regime, as it can be inferred from Figures(9) and (11). Local yielding in the 


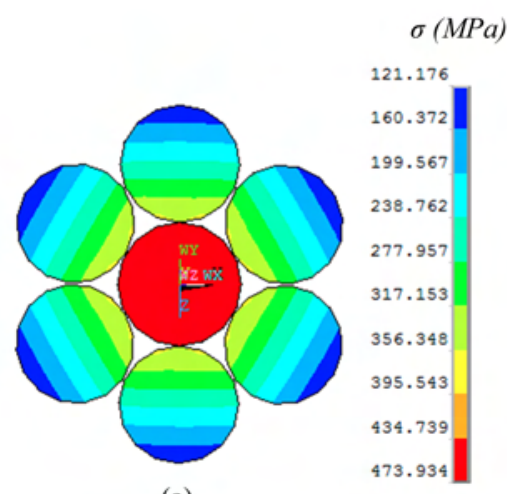

(a)

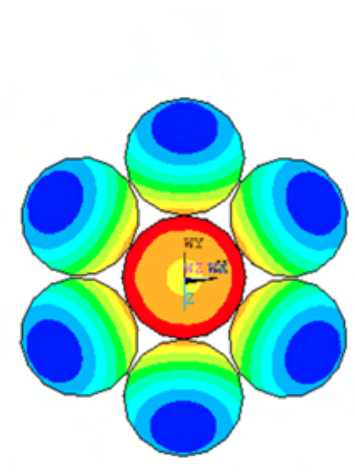

(b)

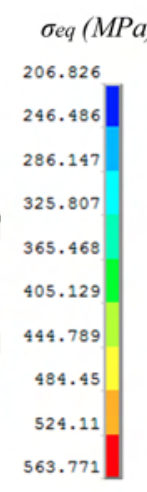

Figure 10: Free-end case. Results of the FE model. Load 21.5 kN. (a) Normal stresses. (b) Von Mises equivalent stresses.

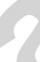

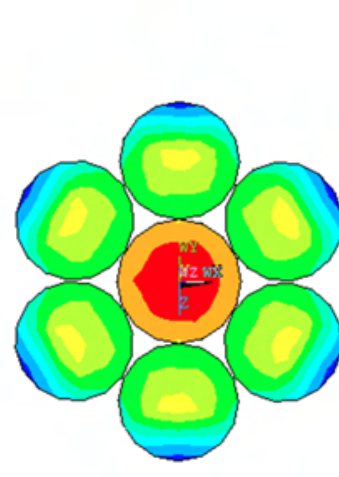

(a)

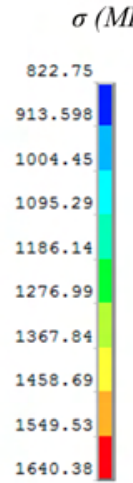

1640.38

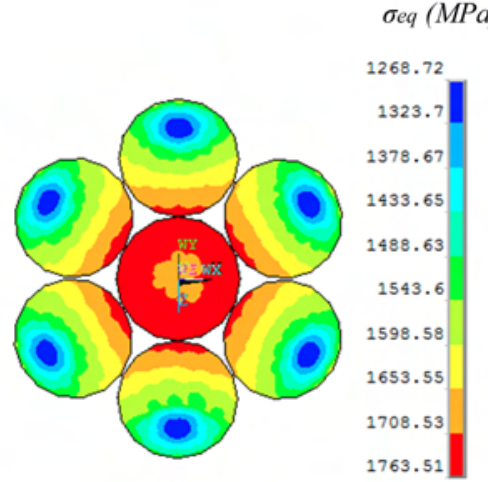

(b)

Figure 11: Free-end case. Results of the FE model. Load 100 kN. (a) Normal stresses. (b) Von Mises equivalent stresses. 

can be clearly appreciated from Figure 11(b). The analytical solution do not capture these local effects (see Figure 9(b)), but yet it delivers both an acceptable estimate of the maximum stresses over the wire cross sections and an excellent prediction of the global strand response curve (Figure 6(a)).

Additional analyses have been carried out to check the accuracy of the proposed analytical formulation against the more rich FE model for different strand constructions. In all the cases, the same geometrical and material properties listed in Table 1 are assumed for the core and external wires. Three different values of lay angle are considered, namely: $5^{\circ}, 10^{\circ}$ and $15^{\circ}$. The highest one is close to the maximum value: $\alpha_{\max }=15.4^{\circ}$, which corresponds to initial circumferential contact conditions and can be calculated through equation (3). Circumferential contact is not considered in the present work, since, as already mentioned in Section 2, typical strand constructions are characterized by interwire clearances. FE models have been generated for each lay angle by considering a $500 \mathrm{~mm}$ length strand, similarly as for the benchmark test case previously studied.

The strand axial force $F_{S}$ is plotted against the axial strain $\varepsilon_{S}$ in Figures 12(a) and 12(b), respectively, for the fixed- and the free-end case. Results of the analytical and FE models are in very good agreement, for both boundary conditions, over the whole range of lay angles herein considered. It's interesting to note that the variations of the lay angle slightly affect the load-strain curve of the strand in the fixed-end case. On the other hand, in the free-end case a variation from $5^{\circ}$ to $15^{\circ}$ of the lay angle leads to a dramatic decreasing of both the initial (elastic) stiffness and the load-bearing capacity of the strand.

Figure (13) shows the relation between the axial load and the reacting torque, in the fixed-end case, for the different values of lay angle herein considered. The relation between axial load and torsional moment is almost linear with decreasing slope for increasing values of the lay angle, i.e.: larger reacting moment are needed to prevent torsional rotations for strands with larger lay angles. A small change of the slope, as already discussed with reference to the previously studied benchmark problem, can be 

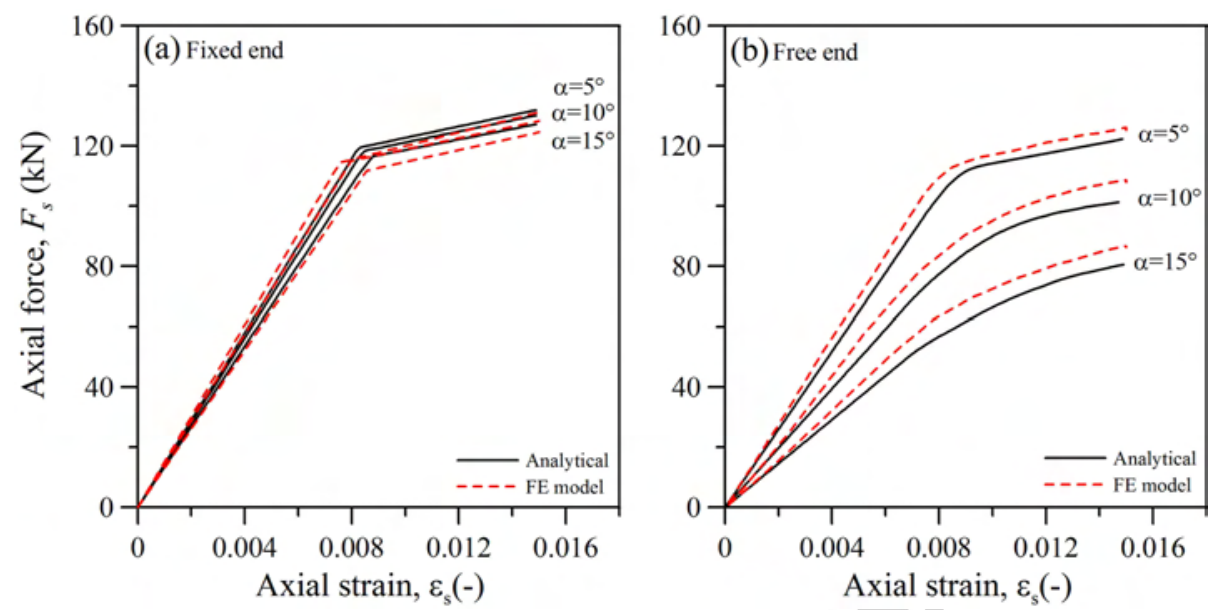

Figure 12: Comparison among the results of the analytical and FE models, for different values of lay angle $\alpha$. Axial force vs. axial strain: (a) fixed-end case; (b) free-end case.

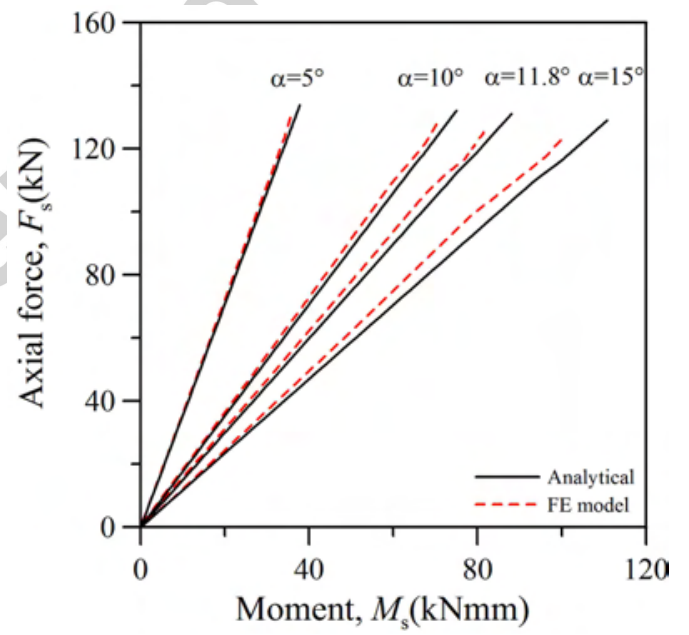

Figure 13: Comparison among the results of the analytical and FE models, for different values of lay angle $\alpha$. Axial force vs. torsional moment in the fixed-end case. 

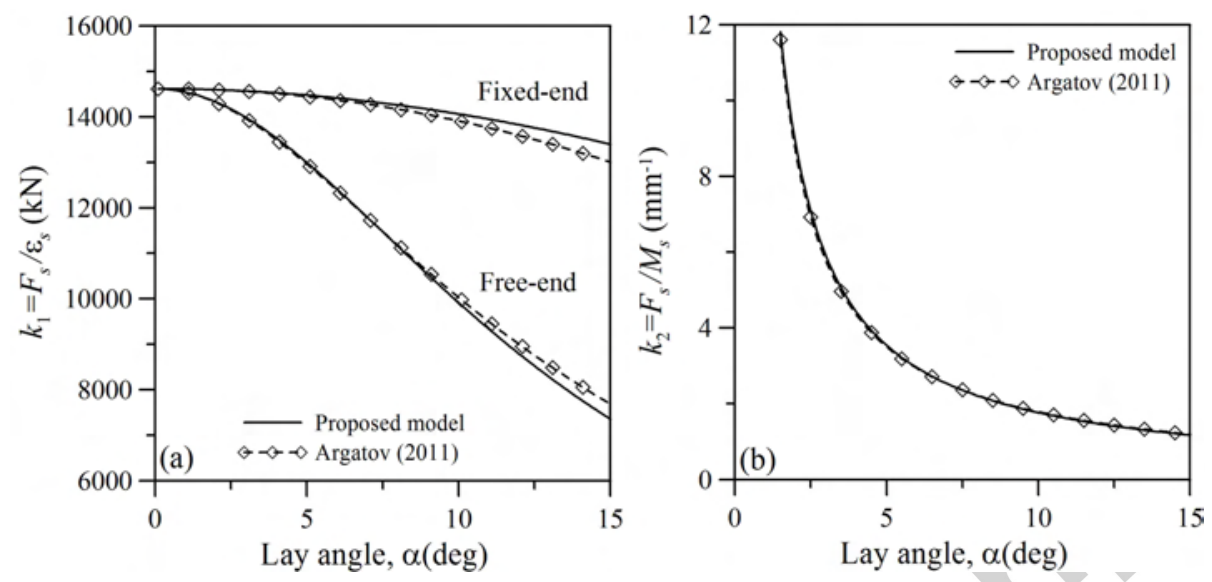

Figure 14: Comparison among the results of different analytical formulations, for different values of lay angle $\alpha$ : (a) direct axial stiffness $k_{1}=\frac{F_{s}}{\varepsilon_{s}}$; (b) coupling parameter $k_{2}=\frac{F_{s}}{M_{s}}$ (fixed-end case).

the value corresponding to the knee of the load-strain curve (see Figure 12(a)). Excellent agreement is observed among the results of the analytical and FE models, with maximum differences on the average slope of the load-torque curve in the order of $5 \%$.

The proposed analytical formulation has been also compared, in the elastic range of deformation, to a recent analytical model developed by Argatov [2]. The latter is based on the linearly elastic curved thin rod theory and on a refined interwire contact model, accounting for the radial contraction of the strand due to both the Poisson effect and the wire flattening.

The direct axial stiffness $k_{1}=\frac{F_{s}}{\varepsilon_{s}}$ predicted by the two different analytical models is plotted in Figure 14(a) as a function of the lay angle $\alpha$. The results are in excellent agreement, with maximum differences in the order of $3 \%$ for the fixed-end case and $4 \%$ for the free-end case. The two models also deliver very close values of the coupling parameter $k_{2}=\frac{F_{s}}{M_{s}}$ (fixed-end case), shown in Figure 14(b) for different values of lay angle. These results lead to the conclusion that, for the cases considered in this work, neglecting the radial contraction of the strand, while simplifying the mechanical formulation still leads to a good estimate of the elastic stiffness parameters of the element. 


\section{Conclusions}

The paper investigates the elastic-plastic behaviour of metallic strands under axial-

425

440

torsional loads, through the combined application of an innovative analytical approach and a three-dimensional (3D) finite element (FE) model.

A new mechanical formulation is developed in this work. The strand is regarded as a composite structural element and each wire is individually modelled as a curved thin rod, which can experience complex stress states due to a combination of axial elongation, torsion and bending. The evolution of plastic deformations in the wires is fully considered, in order to provide an effective engineering tool for the analysis of the strand response under both service as well as ultimate loading conditions. Simple and accurate constitutive equations are derived to relate the coupled axial load and torsional moment of the strand to the work-conjugated axial elongation and torsional curvature.

The performance of the proposed analytical formulation is assessed through comparisons both with experimental and theoretical results from the literature as well as with the numerical predictions of a full $3 \mathrm{D}$ non-linear FE model. The FE model is defined in this work starting from a parametric description of the strand internal geometry, aiming to provide a means for a comparative assessment of the performance of the proposed formulation over a significant range of typical strand construction parameters.

Excellent agreement is found among the experimental results and the proposed analytical and FE models, which allow to describe, both from a qualitative as well as from a quantitative point of view, the evolution of the elastic-plastic response curves of the strand. Also the experimentally observed coupling between the axial force and the torsional moment is very well described by the proposed models. Furthermore, the analytical and FE estimates of the elastic stiffness terms of the strand are shown to be in excellent agreement with both the experimental values and the predictions of well-established linearly elastic literature models. Systematic comparisons among the analytical and FE results are also carried out to show the ability of the proposed mechanical approach in reproducing the local stress state of the wires, both for free as well as for fixed torsional rotations of the strand end sections.

Additional comparisons between the analytical and FE models are then performed, 
to carefully assess the validity of the proposed mechanical formulation over a significant range of strand internal geometries. Within this context, the effects on the strand behaviour of (a) different lay angles, and (b) different torsional boundary conditions are highlighted. In particular, it is found that variations of the lay angle slightly affect the strand load-strain curve whenever torsional rotations of the cross sections are prevented. The reacting moment due to the axial-torsional coupling of the strand, however, can increase significantly for increasing values of the lay angle. Whenever torsional rotations are not constrained, instead, variations of the lay angle can dramatically affect the load-bearing capacity of the strand, which is found to decrease for increasing values of the lay angle.

The applications presented in the paper show that the new mechanical formulation herein proposed can be successfully applied to obtain straightforward analytical estimates of both the elastic stiffness parameters as well as the load-bearing capacity of the strand. The proposed approach, hence, provides some effective tools for engineers involved in strand design, to reduce the need for expensive and time consuming numerical simulations based on non-linear 3D FE models. Moreover, the mechanical framework adopted in this work paves the way for the implementation of the proposed cross sectional constitutive law in finite element formulations suitable for large-scale structural analyses. This can lead to significant refinements of the modelling strategies currently adopted to investigate the post-elastic behaviour of cable structures, which are often based on uni-axial phenomenological constitutive laws.

\section{Appendix A. Evaluation of the wire mechanical curvatures.}

The mechanical curvatures of the wires, $\chi_{w i}(i=1,2,3)$, are defined, consistently with the principle of virtual work, through the expressions first proposed in [11] and re-stated by Foti and Martinelli [8] as:

$$
\chi_{w}=\frac{d \boldsymbol{\psi}_{w}}{d S}+\boldsymbol{\Omega}_{w} \boldsymbol{\psi}_{w}
$$

where: $\chi_{w}$ is a curvature vector defined as $\chi_{w}=\sum_{i=1}^{3} \chi_{w i} \mathbf{f}_{i} ; \boldsymbol{\psi}_{w}$ is a vector collecting the wire cross sectional rotations with respect to the Serret-Frenet unit vectors $\left\{\mathbf{f}_{i}\right\} ; S$ is 
an arc-length coordinate defined on the wire centerline; and $\boldsymbol{\Omega}_{w}$ is a skew-symmetric tensor, whose components $\left(\left[\boldsymbol{\Omega}_{w, i j}\right]\right)$ with respect to the basis $\left\{\mathbf{f}_{i}\right\}$ can be expressed as:

$$
\left[\boldsymbol{\Omega}_{w, i j}\right]=\left[\begin{array}{ccc}
0 & -\kappa & 0 \\
\kappa & 0 & -\tau \\
0 & \tau & 0
\end{array}\right]
$$

The symbols $\kappa$ and $\tau$ in (A.2) denote the initial curvature and torsion of the wire centerline, already defined in Section 2.

The torsional rotation of the strand can be conveniently described, within the strandattached reference system (SRS), by means of the vector: $\psi_{s}\left(x_{1}\right)=\psi_{s 1} \mathbf{e}_{1}$. Then, by assuming that the cross sections of the wires rigidly rotate with the cross section of the strand, the vector $\boldsymbol{\psi}_{w}$ can be evaluated as:

$$
\boldsymbol{\psi}_{w}=\boldsymbol{\Lambda}_{w}^{T} \boldsymbol{\psi}_{s}
$$

where $\boldsymbol{\Lambda}_{w}$ is the rotation tensor giving the orientation of the Serret-Frenet unit vectors $\left\{\mathbf{f}_{i}\right\}$ with respect to the strand SRS (see also equation (2)).

To obtain the wire mechanical curvatures, according to the definition (A.1), it is necessary to derive (A.3) with respect to the arc-length coordinate $S$. Accounting for the differential relation: $d S \cos (\alpha)=d x_{1}$ (see: [7], [8] for more details), the following expression can be easily obtained from (A.3):

$$
\frac{\partial \boldsymbol{\psi}_{w}}{\partial S}=\cos (\alpha) \boldsymbol{\Lambda}_{w}^{T} \boldsymbol{\chi}_{s}+\frac{d \boldsymbol{\Lambda}_{w}^{T}}{d S} \boldsymbol{\Lambda}_{w} \boldsymbol{\psi}_{w}
$$

where $\chi_{s}$ is the strand curvature vector: $\chi_{s}=\chi_{s} \mathbf{e}_{1}$, with: $\chi_{s}=\frac{d \chi_{s}}{d x_{1}}$.

495 The derivative with respect to $S$ of the rotation tensor $\boldsymbol{\Lambda}_{w}$ gives the variation of the orientation of the Serret-Frenet unit vectors along the helicoidal wire centerline. This variation is governed by the well-known Serret-Frenet formulae (see e.g. [21]), which can be expressed as:

$$
\frac{d \boldsymbol{\Lambda}_{w}}{d S}=\boldsymbol{\Lambda}_{w} \boldsymbol{\Omega}_{w}
$$


By recalling the properties of skew-symmetric and rotation tensors, and substituting (A.5) in (A.4), then, the following equation can be obtained:

$$
\frac{\partial \boldsymbol{\psi}_{w}}{\partial S}=\cos (\alpha) \boldsymbol{\Lambda}_{w}^{T} \boldsymbol{\chi}_{s}-\boldsymbol{\Omega}_{w} \boldsymbol{\psi}_{w}
$$

Finally, by substituting (A.6) in (A.1), the mechanical curvatures of the wire can be expressed through the following general equation, firstly presented in [8]:

$$
\chi_{w}=\cos (\alpha) \Lambda_{w}^{T} \chi_{s}
$$

The equation above leads, for the case of pure torsion of the strand (i.e.: $\chi_{s}=\chi_{s} \mathbf{e}_{1}$ ), to the equations $(5 . \mathrm{a}, \mathrm{b}, \mathrm{c})$ introduced in Section 3.

\section{Appendix B. Elastic stiffness coefficients.}

Under the assumption of linearly elastic material, the cross sectional constitutive equations of the strand (see equations $(12 \mathrm{a}, \mathrm{b}))$ can be re-written as:

$$
\begin{aligned}
& F_{s}=E A \varepsilon_{s}+C_{T} \chi_{s} \\
& M_{s}=C_{T} \varepsilon_{s}+C_{T} \chi_{s}
\end{aligned}
$$

where $E A$ and $G J$ denote respectively the direct axial and torsional stiffness of the strand, while $C_{T}$ is the axial-torsional coupling stiffness term.

The following expressions can be obtained for the elastic stiffness coefficients [7]:

$$
E A=E A_{w 0}+6 \cos ^{3}(\alpha) E A_{w}
$$

$$
G J=\frac{E I_{w 0}}{1+v_{0}}+6 \frac{E I_{w}}{1+v}\left(\cos ^{3}(\alpha)+(1+v) \sin ^{2}(\alpha) \cos (\alpha)\right)+6 \sin ^{2}(\alpha) \cos (\alpha) R^{2} E A_{w}
$$

$$
C_{T}=6 \sin (\alpha) \cos ^{2}(\alpha) R E A_{w}
$$


where $E A_{w}$ and $E I_{w}$ are, respectively, the axial and bending stiffness of the wire cross sections, $v$ is the Poisson coefficient of the material, and the subscript " 0 " is adopted to denote quantities referred to the core wire.

Expressions for the ratio $k_{1}=\frac{F_{s}}{\varepsilon_{s}}$ can be easily derived from (B.1) for both the fixed-end $\left(\chi_{s}=0\right)$ as well as the free-end $\left(\chi_{s} \neq 0\right)$ case:

$$
\begin{aligned}
& k_{1}=E A(\text { fixed }- \text { end }) \\
& k_{1}=E A-\frac{C_{T}^{2}}{G J}(\text { free }- \text { end })
\end{aligned}
$$

Finally, in the fixed-end case the ratio $k_{2}=\frac{F_{s}}{M_{s}}$ can be expressed as:

$$
k_{2}=\frac{E A}{C_{T}}
$$

\section{Acknowledgements}

The authors are grateful to Professors Luca Martinelli and Federico Perotti of the Department of Civil and Environmental Engineering of Politecnico di Milano, for their precious advices and support.

\section{References}

[1] Ansys Inc. ANSYS Mechanical User's Guide, Release 15.0. November 2013.

[2] Argatov I. Response of a wire rope strand to axial and torsional loads: Asymptotic modeling of the effect of interwire contact deformations. International Journal of Solids and Structures; 2011; 48; 1413-1423.

[3] Cardou A, Jolicoeur C. Mechanical models of helical strands. Applied Mechanics Review (ASME) 1997; 50; 1-14.

[4] Costello GA. Theory of wire ropes. Springer-Verlag, New York, 1990.

[5] Crisfield MA. Non linear finite element analysis of solids and structures. Volume 
[6] de Luca di Roseto A. Modellazione numerica della risposta ciclica assialetorsionale e flessionale di una fune metallica 1-6. Master Thesis (in Italian), Politecnico di Milano, Milano, 2015.

[7] Foti F. A co-rotational beam element and a refined mechanical model for the nonlinear dynamic analysis of cables. Doctoral Dissertation, Politecnico di Milano, Milano, 2013.

[8] Foti F., Martinelli L. Mechanical modeling of metallic strands subjected to tension, torsion and bending. Theory and Finite Element implementation. International Journal of Solids and Structures 2016; 91; 1-17.

[9] Feyrer K. Wire ropes: tension, endurance, reliability. Springer-Verlag, Berlin Heidelberg, 2007.

[10] Ghoreishi SR, Tanguy M, Cartraud P, Davies P. Validity and limitations of linear analytical models for steel wire strands under axial loading using a 3D FE model. International Journal of Mechanical Sciences; 2007; 49; 1251-1261.

[11] Huang NC. Theories of Elastic Slender Curved Rods. Journal of Applied Mathematics and Physics (ZAMP); 1973; 24; 1-19.

[12] Imrak CE, Erdönmez. On the problem of wire rope model generation with axial load. Mathematical and Computational Applications; 2010; 15; 259-268.

[13] Jiang WG, Yao MS, Walton JM. A concise finite element model for simple straight wire rope strand. International Journal of Mechanical Sciences; 1999; 41; 143-161.

[14] Jiang WG, Henshall JL, Walton JM. A concise finite element model for threelayered straight wire rope strand. International Journal of Mechanical Sciences; 2000; 42; 63-86.

555 [15] Jiang WG, Warby MK, Henshall JL. Statically indeterminate contacts in axially loaded wire strand. European Journal of Mechanics A/Solids; 2008; 27; 69-78. 
[16] Jonatowski JJ, Birnstiel C. Inelastic stiffened suspension space structures. Journal of the Structural Division (ASCE); 1970; 96; 1143-1166.

[17] Judge R, Yang Z, Jones SW, Beattie G. Full 3D finite element modelling of spiral strand cables. Construction and Building Materials; 2012; 35; 452-459.

[18] Kassimali A, Parsi-Feraidoonian H. Strength of cable trusses under combined loads. Journal of Structural Engineering; 1987; 113; 907-924.

[19] Kmet S, Mojdis M. Postelastic analysis of cable trusses. Journal of Structural Engineering; DOI 10.1061/(ASCE)ST.1943-541x.0001227.

[20] Knapp RH. Derivation of a new stiffness matrix for helically armoured cables considering tension and torsion. International Journal for Numerical Methods in Engineering; 1979; 14; 515-529.

[21] Kreyszig E. Differential geometry. Dover Publications, New York, 1991.

[22] Kumar K, Botsis J. Contact stresses in multilayered strands under tension and torsion. Transactions of the ASME; 2001; 68; 432-440.

[23] Lanteigne J. Theoretical estimation of the response of helically armoured cables to tension, torsion and bending. Journal of the Applied Mechanics Division (ASME); 1985; 52; 423-432.

[24] Lemaitre J, Chaboche JL. Mechanics of solid materials, Cambridge University Press, 1990.

[25] Love AEH. A treatise on the mathematical theory of elasticity. Fourth Ed., Dover Publications, New York, 1944.

[26] Raoof M, Kraincanic I. Critical examination of various approaches used for analysing helical cables. Journal of Strain Analysis; 1994; 29; 43-55.

[27] Utting WS, Jones N. The response of wire rope strands to axial tensile loads Part I. Experimental results and theoretical predictions. International Journal of Mechanical Sciences; 1987; 29; 605-619. 
[28] Utting WS, Jones N. The response of wire rope strands to axial tensile loads

Part II: comparisons of experimental results and theoretical predictions. International Journal of Mechanical Sciences; 1987; 29; 621-636.

[29] Velinsky SA, Anderson GL, Costello GA. Wire rope with complex cross section. Journal of Engineering Mechanics (ASCE); 1984; 110; 380-391.

[30] Yu Y, Chen Z, Liu H, Wang, X. Finite element study of behavior and interface force conditions of seven-wire strand under axial and lateral loading. Construction and Building Materials; 2014; 66; 10-18. 